\title{
Endodontic Treatment
}

National Cancer Institute

\section{Source}

National Cancer Institute. Endodontic Treatment. NCI Thesaurus. Code C52575.

Dental procedures to treat disease or injury to the tooth pulp, and may include palliative treatment. 